\title{
Inulin and fructo-oligosaccharides have divergent effects on colitis and commensal microbiota in HLA-B27 transgenic rats
}

\author{
Petya T. Koleva ${ }^{1,2}$, Rosica S. Valcheva ${ }^{1}$, Xu Sun ${ }^{1}$, Michael G. Gänzle ${ }^{2 *}$ and Levinus A. Dieleman ${ }^{1}$ \\ ${ }^{1}$ Centre of Excellence for Gastrointestinal Inflammation and Immunity Research, University of Alberta, Edmonton, \\ AB, Canada T6G $2 \times 8$ \\ ${ }^{2}$ Department of Agricultural, Food and Nutritional Science, University of Alberta, 4-10 Ag/For Centre, Edmonton, \\ AB, Canada T6G 2P5 \\ (Submitted 25 July 2011 - Final revision received 30 November 2011 - Accepted 30 November 2011 - First published online 16 January 2012)
}

\section{Abstract}

Modulation of intestinal microbiota by non-digestible carbohydrates may reduce inflammation in inflammatory bowel disease (IBD). The aim of the present study was to assess the effects of inulin and fructo-oligosaccharides (FOS) on intestinal microbiota and colitis in HLA-B27 transgenic rats, a well-validated rodent model for IBD. In this study, 4-week-old rats were fed $8 \mathrm{~g} / \mathrm{kg}$ body weight inulin or FOS for 12 weeks, or not. Faeces were collected at 4 and 16 weeks of age; and caecal samples were collected at necropsy. The effects of inulin and FOS on chronic intestinal inflammation were assessed using a gross gut score, histology score and levels of mucosal IL-1 $\beta$. Intestinal microbiota were characterised by quantitative PCR and denaturing gradient gel electrophoresis. Colitis was significantly reduced in all FOS-fed rats compared to the control diet, whereas inulin decreased chronic intestinal inflammation in only half the number of animals. Quantitative analysis of caecal microbiota demonstrated that inulin increased the numbers of total bacteria and the Bacteroides-PrevotellaPorphyromonas group, FOS increased bifidobacteria, and both fructans decreased Clostridium cluster XI. In the faecal samples, both inulin and FOS decreased total bacteria, Bacteroides-Prevotella-Porphyromonas group, and Clostridium clusters XI and XIVa. FOS increased Bifidobacterium spp., and mediated a decrease of gene copies of Enterobacteriaceae and Clostridium difficile toxin B in faeces. SCFA concentrations in the faecal and caecal samples were unaffected by the diets. In conclusion, FOS increased the abundance of Bifidobacterium spp., whereas both fructans reduced Clostridium cluster XI and $C$. difficile toxin gene expression, correlating with a reduction of chronic intestinal inflammation.

Key words: Colitis: Fructo-oligosaccharides: Inulin: Denaturing gradient gel electrophoresis: Quantitative PCR: Clostridium difficile

Inflammatory bowel disease (IBD), encompassing ulcerative colitis (UC) and Crohn's disease, is a group of chronic intestinal disorders associated with uncontrolled inflammation within the gastrointestinal $\operatorname{tract}^{(1)}$. The pathogenesis of IBD is attributed to an interaction of genetic, immune and environmental factors ${ }^{(1)}$. Intestinal micro-organisms and their products play an important role in the initiation and perpetuation of chronic intestinal inflammation ${ }^{(2)}$. Inflammation occurs in areas with the highest number of luminal bacteria ${ }^{(3)}$. Broadspectrum antibiotics ${ }^{(4)}$ and surgical diversion of the faecal stream can prevent disease recurrence in Crohn's disease ${ }^{(5)}$. Moreover, microbial imbalance, called 'dysbiosis', is observed in IBD patients ${ }^{(6)}$. IBD patients have greater numbers of mucosa-associated intestinal bacteria compared to non-IBD controls $^{(7)}$. A reduced abundance of Bacteroidetes and Firmicutes species and an increase of virulent Escherichia coli were observed in the mucosa-associated microbiota of IBD patients ${ }^{(7-10)}$. The role of microbiota in IBD was further confirmed in animal models for IBD. HLA-B27/human $\beta 2$ microglobulin transgenic (TG) rats raised under specific pathogen-free environment spontaneously develop chronic colitis mimicking IBD, and arthritis. However, these TG rats raised in germ-free conditions fail to develop inflammation, but introduction of (specific) bacteria induces colitis ${ }^{(4,11,12)}$. Similarly, other rodent models of colitis remain disease-free in the absence of bacteria ${ }^{(13)}$.

Modification of the gastrointestinal microbiota may restore the balance of the host bacteria and reduce inflammation.

Abbreviations: DGGE, denaturing gradient gel electrophoresis; DP, degree of polymerisation; DSS, dextran sodium sulphate; FOS, fructo-oligosaccharides; GGS, gross gut score; IBD, inflammatory bowel disease; PC, principal component; qPCR, quantitative PCR; TG, transgenic; UC, ulcerative colitis 
Non-digestible carbohydrates such as $\beta$-fructans stimulate the growth of specific resident bacteria in the gut and thus allow manipulation of host microbiota. Inulin and fructo-oligosaccharides (FOS) are linear $\beta(2 \rightarrow 1)$ linked fructans. Inulin has a degree of polymerisation (DP) between 10 and 60, whereas FOS has a DP varying between 2 and 10. Dietary inulin and FOS are not hydrolysed by mammalian enzymes, but are readily fermented by the bacterial community in the caecum and colon, and favour the growth of intestinal bifidobacteria $^{(14,15)}$. These $\beta$-fructans occur at high levels in plants such as chicory, asparagus, leek, onions, garlic and Jerusalem artichokes, and are also produced by Lactobacillus spp. in cereal fermentations ${ }^{(16,17)}$.

Studies in animal models for IBD indicate that a combination of inulin and FOS reduce chronic intestinal inflammation. Treatment of HLA-B27 TG rats with inulin and FOS reduced inflammation in conjunction with an increase of caecal Lactobacillus spp. and Bifidobacterium spp. ${ }^{(18,19)}$. Inulin and FOS alone or in combination with two strains of Bifidobacterium infantis also reduced inflammation in dextran sodium sulphate (DSS)-induced colitis in rats ${ }^{(20)}$. However, FOS exacerbated DSS-induced colitis in mice fed a purified diet ${ }^{(21)}$. Small clinical studies in patients with active UC reported reduced colonic inflammation after oral therapy with Bifidobacterium longum in combination with the prebiotics inulin and $\mathrm{FOS}^{(22)}$, whereas a reduction of the inflammatory faecal marker calprotectin was reported in active UC patients administered inulin plus FOS ${ }^{(23)}$. However, the use of FOS plus inulin mixture was ineffective in a large randomised, placebo-controlled trial in patients with mild-to-moderately active Crohn's disease ${ }^{(24)}$. Whereas studies in animal models and some of the clinical trials indicate that prebiotics show promise in the treatment or prevention of IBD, the divergent outcomes of studies clearly demonstrate that additional data on the mechanisms of action of non-digestible carbohydrates are required before these can be recognised as a valid tool in the management of IBD.

Inulin and FOS differ in their effects on caecal, colonic and faecal microbiota of rats that were colonised with human microbiota $^{(25)}$. Likewise, dietary $\beta$-glucans differing in their molecular weight exerted different effects on the intestinal microbiota in pigs ${ }^{(26)}$. However, past studies on dietary intervention to prevent colitis in rodent models provided only a partial characterisation of intestinal microbiota ${ }^{(18,20)}$. Moreover, no studies have been performed using $\beta$-fructans with different DP in a well-validated, spontaneous colitis model. It was therefore the aim of the present study to compare the effect of inulin and FOS on intestinal inflammation and to determine how changes in the profile of caecal and faecal microbiota correlate with colitis reduction in HLA-B27 TG rats, the model in which this prebiotic mixture was previously shown to be beneficial. Inflammation was assessed by a validated gross gut score (GGS), histology score, and by quantification of pro-inflammatory mucosal IL1- $\beta$ concentration.

\section{Materials and methods}

\section{Experimental design and sample collection}

HLA-B27 TG rats, a validated colitis model, were used in this study. Animals were randomly assigned to three different treatment groups: (1) commercial standard diet as a control (5053 PicoLab® Rodent Diet 20; Lab Diet, Inc.); (2) FOS (average DP 4, Orafti P-95; Raffinerie Tirlemontoise); (3) inulin (average DP 25, Orafti HP). Then, $8 \mathrm{~g} / \mathrm{kg}$ body weight of the respective fructans were mixed with the standard diet, a dose previously found to be optimal for its colitis-reducing effects $^{(27)}$. Feed was provided ad libitum and the average feed consumption of the rats was $20 \mathrm{~g}$ of standard diet/d. The body weight of the animals was measured every 2 weeks and the fructan addition to the diet was adjusted according to the body weight. The number of animals included in the study was as follows: control group - thirteen females and nine males, FOS group - three females and nine males and inulin group - seven females and eight males. Treatment started at 4 weeks of age (before colitis occurred) and continued until 16 weeks of age. Faecal samples were collected from each group at 4 and 16 weeks of age. All rats were euthanised at 16 weeks of age. At necropsy, caecal and colonic tissues and their contents were taken for histology, quantification of mucosal IL-1 $\beta$, as well as for microbiota analysis. All samples were immediately frozen and stored at $-80^{\circ} \mathrm{C}$. Animal trials were approved by the University of Alberta Animal Policy and Welfare Committee in accordance with the Canadian Council on Animal Care guidelines.

\section{Assessment of treatment effects on inflammation}

Caecal and colon tissues were fixed and stained with haematoxylin and eosin, as previously described ${ }^{(4)}$. Blinded evaluation of caecal and colonic microscopic inflammation was performed using a validated histology score ranging from 0 to $4^{(4)}$. Histology score included the following parameters: (1) number of inflammatory and goblet cells, (2) mucosal thickening, (3) infiltration of submucosal cells and (4) destruction of the architecture of the intestinal epithelium. The degree of macroscopic inflammation was further assessed using a validated GGS based on a scale from 0 to 4 , as previously described $^{(4)}$. Caecal inflammation evaluated by GGS included the criteria (1) number of caecal nodules, (2) severity of mesenteric contractions, (3) severity of adhesions and (4) extent of caecal wall thickening. The mucosal IL1- $\beta$ concentration in the caecal and colonic homogenates was quantified by a rat-specific IL1- $\beta$ ELISA $^{(11)}$, using a commercial DuoSet ELISA Development System kit (R\&D Systems, Inc.). Recombinant rat IL-1 $\beta$ with known concentration was used as standard for the ELISA assay and 2-fold dilutions of the standard were run on each plate. Results were calculated using total protein for normalisation of the targeted cytokine measurement and presented as $\mu \mathrm{g}$ IL- $1 \beta / \mathrm{g}$ protein.

\section{Genomic DNA extraction and quantitative $P C R$ analysis}

Bacterial DNA was extracted from the caecal and faecal samples using a QIAamp DNA Stool Mini Kit (Qiagen, Inc.). 
Quantity and quality of DNA were checked on a NanoDrop spectrophotometer system ND-1000, version 3.3.0 (Thermo Fisher Scientific, Inc.). Before PCR analysis, samples were diluted to contain comparable DNA concentrations.

Quantitative PCR (qPCR) was performed on a 7500 Fast Real-Time PCR System (Applied Biosystems). The primers and probes based on 16S rRNA gene sequences were chosen to target total bacteria, Bacteroides-Prevotella-Porphyromonas spp., the dominant group of Gram-negative bacteria, Clostridium clusters IV and XIVa, the main Gram-positive bacterial groups in intestinal microbiota, as well as clostridial clusters I and XI, which include pathogens (Table 1). Genes encoding Clostridium difficile toxin $\mathrm{B}$ and Clostridium perfringens $\alpha$ toxin were quantified by qPCR to specifically identify toxin-producing organisms in these clusters (Table 1). Microbiota analysis further included the Enterobacteriaceae family, which also includes pathogens, and bacteria known to have beneficial effect on the host such as Bifidobacterium spp. and Lactobacillus-Pediococcus-Leuconostoc-Weissella spp. (Lactobacillus group; Table 1). Samples were analysed in duplicate in MicroAmp Fast Optical ninety-six-well reaction plates capped with MicroAmp Optical Adhesive Film (Applied Biosystems). The PCR reaction mixtures consisted of $12.5 \mu \mathrm{l}$ Fast SYBR Green Master Mix (Applied Biosystems), $0 \cdot 4 \mu \mathrm{M}$ of each primer (Table 1), $2 \mu$ l of template DNA of caecal or faecal samples and sterile Milli-Q water to final volume of $25 \mu \mathrm{l}$. The cycling programme was as follows: initial denaturation at $95^{\circ} \mathrm{C}$ for $5 \mathrm{~min}$; forty cycles of $95^{\circ} \mathrm{C}$ for $15 \mathrm{~s}$, primer annealing at the optimal temperatures (Table 1) for $30 \mathrm{~s}$, and extension at $72^{\circ} \mathrm{C}$ for $30 \mathrm{~s}$. Melting curves were obtained by a stepwise increase of the temperature from 60 to $95^{\circ} \mathrm{C}$ (at $10 \mathrm{~s} / 0 \cdot 5^{\circ} \mathrm{C}$ ). Melting-curve data were analysed to verify amplification of the correct targeted PCR products. For quantification with the gene-specific primers and the groupspecific primers for Clostridium cluster XI, TaqMan assays were performed. Amplifications were carried out in a total volume of $25 \mu \mathrm{l}$, containing $12.5 \mu \mathrm{l}$ TaqMan Universal PCR Master Mix, No AmpErase UNG (Applied Biosystems), 0 $4 \mu \mathrm{M}$ of each primer (Table 1), $1 \mu$ l of TaqMan probe (100 nM), $2 \mu \mathrm{l}$ of template DNA of caecal or faecal samples and $7 \cdot 5 \mu \mathrm{l}$ sterile Milli-Q water. These amplification conditions were as follows: one cycle at $50^{\circ} \mathrm{C}$ for $2 \mathrm{~min}$; one cycle at $95^{\circ} \mathrm{C}$ for $10 \mathrm{~min}$; forty-five cycles of $95^{\circ} \mathrm{C}$ for $20 \mathrm{~s}$, primer annealing at the optimal temperatures (Table 1) for $30 \mathrm{~s}$, and extension at $72^{\circ} \mathrm{C}$ for $45 \mathrm{~s}$. Fluorescent emission was measured at the extension step for the SYBR Green assays and at the primer annealing step for TaqMan assays. For generation of standard curves, 10-fold serial dilutions of purified and quantified PCR products were used. The standard curves of the individual qPCR assays were obtained by PCR using primers listed in Table 1 and genomic DNA isolated from Clostridium perfringens ATCC 13124, Clostridium difficile 75, or DNA extracted from the faecal samples. Individual reactions of the standard curves were run in duplicate on each plate for the respective bacterial

Table 1. Primers and PCR conditions

\begin{tabular}{|c|c|c|c|c|}
\hline Target group/gene & Oligonucleotide sequence $\left(5^{\prime}-3^{\prime}\right)$ & $T_{\mathrm{m}}\left({ }^{\circ} \mathrm{C}\right)$ & Product size (bp) & Reference or source \\
\hline Domain bacteria (total bacteria) & $\begin{array}{l}\text { F: CGGYCCAGACTCCTACGGG } \\
\text { R: TTACCGCGGCTGCTGGCAC }\end{array}$ & 63 & 200 & Lee et al. ${ }^{(50)}$ \\
\hline $\begin{array}{l}\text { Lactobacillus-Pediococcus- } \\
\quad \text { Leuconostoc group }\end{array}$ & $\begin{array}{l}\text { F: AGCAGTAGGGAATCTTCCA } \\
\text { R: CACCGCTACACATGGAG } \\
\text { GC clamp-CACCGCTACACATGGAG* }\end{array}$ & 63 & 341 & $\begin{array}{l}\text { Walter et al. }{ }^{(51)} \\
\text { Heilig et al. }{ }^{(2)} \\
\text { This study }\end{array}$ \\
\hline $\begin{array}{l}\text { Bacteroides-Prevotella- } \\
\text { Porphyromonas group }\end{array}$ & $\begin{array}{l}\text { F: GGTGTCGGCTTAAGTGCCAT } \\
\text { R: CGGAYGTAAGGGCCGTGC } \\
\text { GC clamp-CGGAYGTAAGGGCCGTGC* }\end{array}$ & 60 & 140 & $\begin{array}{l}\text { Rinttila et al. }{ }^{(53)} \\
\text { This study }\end{array}$ \\
\hline Enterobacteriaceae family & $\begin{array}{l}\text { F: CATTGACGTTACCCGCAGAAGAAGC } \\
\text { R: CTCTACGAGACTCAAGCTTGC }\end{array}$ & 63 & 195 & Bartosch et al. ${ }^{(54)}$ \\
\hline Bifidobacterium spp. & $\begin{array}{l}\text { F: TCGCGTCYGGTGTGAAAG } \\
\text { R: CCACATCCAGCRTCCAC }\end{array}$ & 60 & 243 & Rinttila et al. ${ }^{(53)}$ \\
\hline & $\begin{array}{l}\text { g-Bifid F: CTCCTGGAAACGGGTGG } \\
\text { g-Bifid R GC: GC clamp-GGTGTTCTTCCCGATATCTACA† }\end{array}$ & 65 & 596 & Matsuki et al. ${ }^{(55)}$ \\
\hline Clostridium cluster I & $\begin{array}{l}\text { F: ATGCAAGTCGAGCGAKG } \\
\text { R: TATGCGGTATTAATCTYCCTTT }\end{array}$ & 58 & 120 & Rinttila et al. ${ }^{(53)}$ \\
\hline Clostridium cluster IV & $\begin{array}{l}\text { F: GCACAAGCAGTGGAGT } \\
\text { R: CTTCCTCCGTTTTGTCAA } \\
\text { GC clamp-CTTCCTCCGTTTTGTCAA* }\end{array}$ & 60 & 239 & $\begin{array}{l}\text { Matsuki et al. }{ }^{(56)} \\
\text { This study }\end{array}$ \\
\hline Clostridium cluster XIVa & $\begin{array}{l}\text { F: AAATGACGGTACCTGACTAA } \\
\text { R: CTTTGAGTTTCATTCTTGCGAA }\end{array}$ & 58 & $438-441$ & Matsuki et al. ${ }^{(55)}$ \\
\hline Clostridium cluster XI & $\begin{array}{l}\text { F: ACGCTACTTGAGGAGGA } \\
\text { R: GAGCCGTAGCCTTTCACT } \\
\text { FAM-GTGCCAGCAGCCGCGGTAATACG-BHQ }\end{array}$ & 58 & 139 & Song et al. ${ }^{(57)}$ \\
\hline Clostridium perfringens $\alpha$ toxin & $\begin{array}{l}\text { F: GCTAATGTTACTGCCGTTGA } \\
\text { R: CCTCATTAGTTTTGCAACC } \\
\text { 6FAM-GCGCAGGACATGTTAAGTTTG-TAMRA }\end{array}$ & 55 & 109 & $\begin{array}{l}\text { Messelhäusser } \\
\quad \text { et al. }\end{array}$ \\
\hline Clostridium difficile toxin B & $\begin{array}{l}\text { 398CLDs: GAAAGTCCAAGTTTACGCTCAAT } \\
\text { 399CLDas: GCTGCACCTAAACTTACACCA } \\
\text { FAM-ACAGATGCAGCCAAAGTTGTTGAATT-TAMRA }\end{array}$ & 58 & 177 & $\begin{array}{l}\text { Van den Berg } \\
\text { et al. }{ }^{(59)}\end{array}$ \\
\hline
\end{tabular}

$T_{\mathrm{m}}$, melting temperature; $\mathrm{F}$, forward; $\mathrm{R}$, reverse.

* GC clamp sequence - CGCCCGGGGCGCGCCCCGGGCGGGGCGGGGGCACGGGGGG.

†GC clamp sequence - CGCCCGCCGCGCCCCGCGCCCGGCCCGCCGCCCCCGCCCC. 
group. The detection limit was $10^{2}$ copy numbers/g caecal content or faeces for the group-specific primers and primers for $C$. difficile toxin $\mathrm{B}$ and $C$. perfringens $\alpha$ toxin.

\section{Analysis of faecal microbiota using PCR-denaturing gradient gel electrophoresis}

A total of four sets of group-specific primers targeting regions of the bacterial $16 \mathrm{~S}$ rRNA gene were used to investigate the diversity of Lactobacillus group, Bifidobacterium spp., Bacteroides group and Clostridium cluster IV by PCR-DGGE. PCR specific for bifidobacteria was performed using primers g-Bifid F and g-Bifid R-GC (Table 1) in a Gene Amp PCR System 9700 (Applied Biosystems). To assess diversity in the Lactobacillus group, Bacteroides group, and Clostridium cluster IV in faecal microbiota by DGGE, the respective groupspecific primer sets were employed at the appropriate annealing temperature (Table 1). PCR products were then used as templates in a second PCR, performed with the same primer pairs with attached GC clamp to the $5^{\prime}$ end of the reverse primer (Table 1). Amplicons were checked by electrophoresis in $2 \%(\mathrm{w} / \mathrm{v})$ agarose gel before DGGE analysis.

PCR fragments were analysed by DGGE with a DCode Universal Mutation Detection System (Bio-Rad) on a $6 \%$ (w/v) polyacrylamide gel (37.5:1 acrylamide-bisacrylamide). Bifidobacterium-specific amplicons were separated in 40-70\% denaturing gradient (100\% corresponds to $7 \mathrm{~m}$-urea and $40 \%$ (w/v) formamide). Amplicons obtained with primers targeting Lactobacillus and Bacteroides groups, and Clostridium cluster IV were separated in a denaturing gradient from $30 \%$ to $55 \%$. Electrophoresis was performed in buffer containing $40 \mathrm{~mm}$-Tris, $20 \mathrm{~mm}$-acetic acid, $1 \mathrm{~mm}$-EDTA at a constant voltage of $130 \mathrm{~V}$ and a temperature of $60^{\circ} \mathrm{C}$ for $4 \mathrm{~h}$. Gels were stained with SYBR Safe $1 \times$ solution (Invitrogen) for $1 \mathrm{~h}$ and $30 \mathrm{~min}$ and photographed by UV transillumination. DGGE profiles were compared using Bionumerics software (version 4.01, Applied Maths) and similarities were expressed based on Pearson correlation coefficients. All gels were normalised using a reference sample with bands distributed throughout the whole gel.

\section{DNA sequencing}

A total of five samples were randomly chosen and amplified in duplicates with group-specific primers for bifidobacteria (Table 1). Samples were analysed by DGGE, and the DGGE bands obtained with primers g-Bifid $F$ and g-Bifid R-GC were sequenced by service of Macrogen. Sequences were compared to those in the Ribosomal Database Project (rdp.cme.msu.edu/seqmatch). The GenBank accession numbers for the sequences are HQ283419, HQ283420, HQ283421, HQ283422 and HQ268606.

\section{Analysis of SCFA}

Caecal contents and faeces $(100 \mathrm{mg})$ were mixed with $300 \mu \mathrm{l}$ water, vortexed vigorously and centrifuged for $15 \mathrm{~min}$ at $20800 \mathrm{~g}$. The supernatant $(100 \mu \mathrm{l})$ was mixed with $300 \mu \mathrm{l}$
$7 \%$ perchloric acid, incubated at $4^{\circ} \mathrm{C}$ overnight and the precipitates were removed by centrifugation for $5 \mathrm{~min}$ at 20800 g. SCFA concentration was determined using HPLC (Agilent 1200 Series; Agilent Technologies, Inc.) equipped with an Aminex HPX-87H column (Bio-Rad Laboratories). The column was eluted with $5 \mathrm{~mm}-\mathrm{H}_{2} \mathrm{SO}_{4}$ at a temperature of $70^{\circ} \mathrm{C}$ and a flow rate of $0.4 \mathrm{ml} / \mathrm{min}$. Detection of the SCFA was achieved employing UV $(210 \mathrm{~nm})$ and refractive index detectors. External standards were used to calculate the concentration of the SCFA.

\section{Statistical analysis}

Data analysis was performed using the mixed procedure (PROC MIXED) of the Statistical Analysis Systems (SAS Insti-
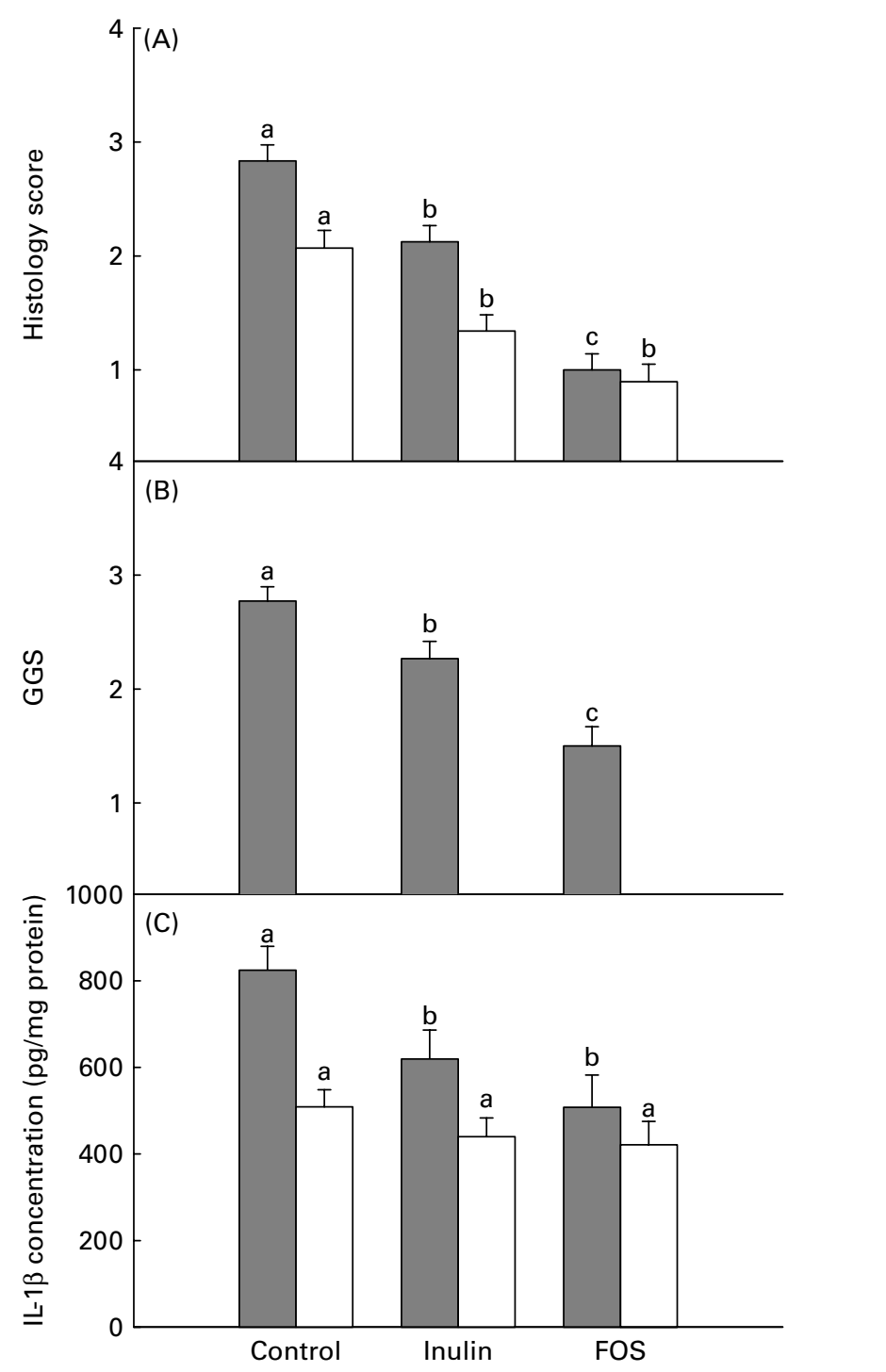

Fig. 1. (A) Histology score, (B) gross gut score (GGS) and (C) IL-1 $\beta$ concentration of caecal $(\square)$ and colonic ( $\square$ ) tissue samples collected from HLA-B27 transgenic rats. The rats were either treated with inulin or fructooligosaccharides (FOS), or not. Values are least-square means, with their standard errors represented by vertical bars. ${ }^{a, b, c}$ Least-square mean values (obtained from the same tissue) with unlike letters were significantly different $(P \leq 0.05)$ 
tute, Inc.). The differences in faecal microbiota and SCFA between groups were evaluated employing repeatedmeasures ANOVA. There were two fixed effects - time and treatment, whereas the random variation was individual rats. The variance and covariance associated with the responses over time were assumed by compound symmetry structure. To detect differences between groups, caecal bacterial populations, SCFA and caecal tissue inflammation were analysed using randomised block design, in which treatments were fixed effects and animals were random effects. Df were estimated by the Kenward-Rogers method and the probability of difference was used to test differences between leastsquare means of different treatments. Results are expressed as least-square means with their standard errors. A $P$ value of $<0.05$ was considered statistically significant.

Principal component analysis and linear discriminant analysis were performed using JMP software (version 8.0.1, SAS Institute, Inc.). The loading plot is a graphical representation of the amount of variation within the data set and shows the correlation of the individual variables of the first two principal components (PC1 and PC2). The correlations between mucosal inflammation and bacterial populations were assessed by Spearman's correlation test using GraphPad Prism version 5.00 (GraphPad Software)

\section{Results \\ Effect of inulin and fructo-oligosaccharides on chronic intestinal inflammation}

The evaluation of mucosal IL-1 $\beta$ concentration and histology scores indicated that the caecum was more inflamed in comparison with colonic tissue in rats on a control diet (Fig. 1(A)-(C)). Histology scores and GGS showed that inulin and FOS reduced inflammation in the caecum and colon (Fig. 1(A) and (B)). The concentration of the proinflammatory cytokine IL- $1 \beta$ was decreased in caecal tissue of animals treated with inulin $(P=0.024)$ or FOS $(P<0.001)$ $v$. control rats, further confirming a protective effect on intestinal inflammation by $\beta$-fructans treatment (Fig. 1(C)). However, GGS and histology scores indicated that the intestinal inflammation in FOS-treated rats was less severe compared to inulin-treated animals (Fig. 1(A) and (B)).

\section{Quantification of bacterial populations by quantitative $P C R$}

To determine the dietary impact of the different treatments on caecal and faecal microbiota of HLA-B27 TG rats, qPCR was performed (Figs. 2 and 3). Faecal and caecal samples were analysed to allow the comparison of samples from the same

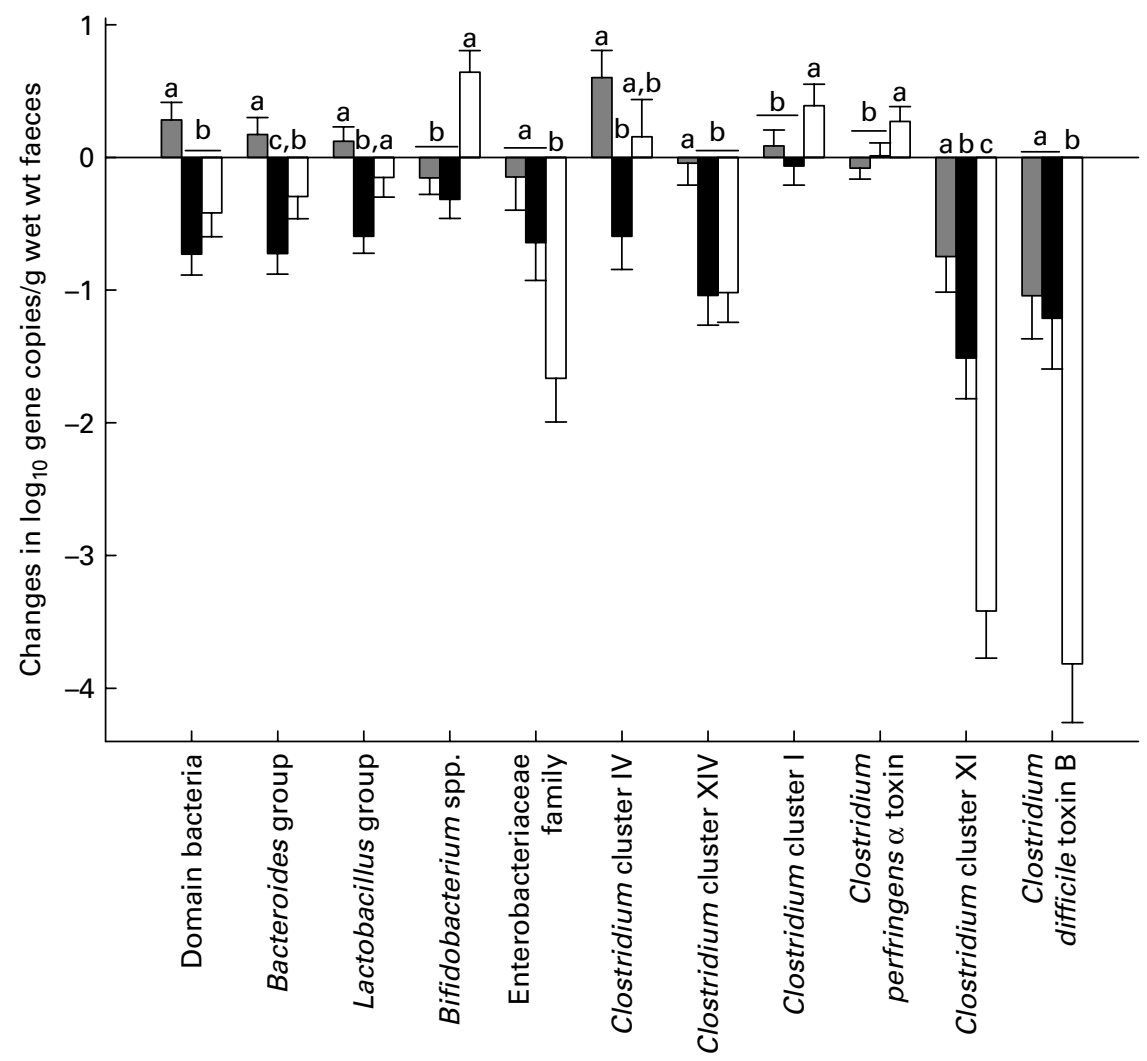

Fig. 2. Quantification of bacterial populations of faecal samples ( $\square$, control; $\mathbf{\square}$, inulin; $\square$, fructo-oligosaccharides) collected from HLA-B27 transgenic rats before and at the end of the fibre treatments. Values are change in $\log _{10}$ copy numbers of $16 \mathrm{~S}$ rDNA (week 16 - week 4 ) least-square means, with their standard errors represented by vertical bars. ${ }^{\mathrm{a}, \mathrm{b}, \mathrm{c}}$ Least-square mean values (obtained with the same primer pairs) with unlike letters were significantly different $(P \leq 0.05)$. 
animal over time (faecal samples, Fig. 2) as well as the analysis from the intestinal site with the highest degree of inflammation (caecal samples, Fig. 3). Studying faecal microbiota, treatment with both fructans decreased the numbers of total bacteria and the Bacteroides group. Dietary intervention with fructans reduced Clostridium cluster XI and cluster XIVa by more than one $\log$ (Fig. 2). FOS treatment increased the numbers of bifidobacteria and Clostridium cluster I $(P<0.001)$; however, FOS-fed animals also showed significantly decreased copy numbers of Enterobacteriaceae $(P=0.023)$ and $C$. difficile toxin $\mathrm{B}(P<0 \cdot 001)$. Inulin specifically mediated the reduction of the Lactobacillus group $(P=0.03)$ and clostridial cluster IV $(P<0.001)$. Animals harboured high copy numbers of $16 \mathrm{~S}$ rRNA genes of organisms belonging to the clostridial cluster I. This cluster comprises commensal fibrolytic and butyrateproducing bacteria as well as toxinogenic species such as $C$. perfringens ${ }^{(28)}$. The low abundance of gene copy numbers of $C$. perfringens $\alpha$ toxin indicates very low numbers of toxinogenic $C$. perfringens. However, gene copy numbers of $C$. difficile toxin B were equivalent to the copy numbers of $16 \mathrm{~S}$ rRNA genes of Clostridium cluster XI, which demonstrated that $C$. difficile was the main representative of that clostridial cluster (Figs. 2 and 3).

The effects of inulin and FOS treatments on the caecal microbiota were less pronounced compared to their effects on the faecal microbiota (Fig. 3). Compared to caecal samples from control animals, inulin increased the numbers of $16 \mathrm{~S}$
rRNA gene copies of total bacteria $(P=0.006)$ and organisms from the Bacteroides-Prevotella-Porphyromonas group ( $P=0.008)$, but reduced organisms in Clostridium cluster XI $(P<0.001)$ (Fig. 3). FOS treatment increased the numbers of caecal bifidobacteria by one $\log (P<0.001)$, whereas Clostridium cluster IV $(P=0.015)$ and Clostridium cluster XI $(P<0 \cdot 001)$ were decreased $v$. rats in the control group. The numbers of caecal bifidobacteria and organisms belonging to clostridial clusters I and IV in FOS-treated animals were also significantly different in comparison to rats treated with inulin.

\section{PCR-denaturing gradient gel electrophoresis profiles}

To determine whether quantitative changes in intestinal microbiota were accompanied by qualitative changes, PCRDGGE analysis was performed using primers targeting four phylogenic groups whose abundance was altered by dietary intervention with fructans. The cluster analysis for DGGEprofiles obtained with group-specific primers targeting the Bacteroides group is shown in Fig. 4. DGGE patterns were separated in two main clusters. The upper cluster mainly consisted of inulin- and FOS-treated animals, whereas the lower cluster contained mainly control animals (Fig. 4). This result indicates that diet-induced changes in the abundance were indeed associated with qualitative changes in composition of bacterial taxa in the Bacteroides group. However, patterns generated with primers specific for the Lactobacillus group

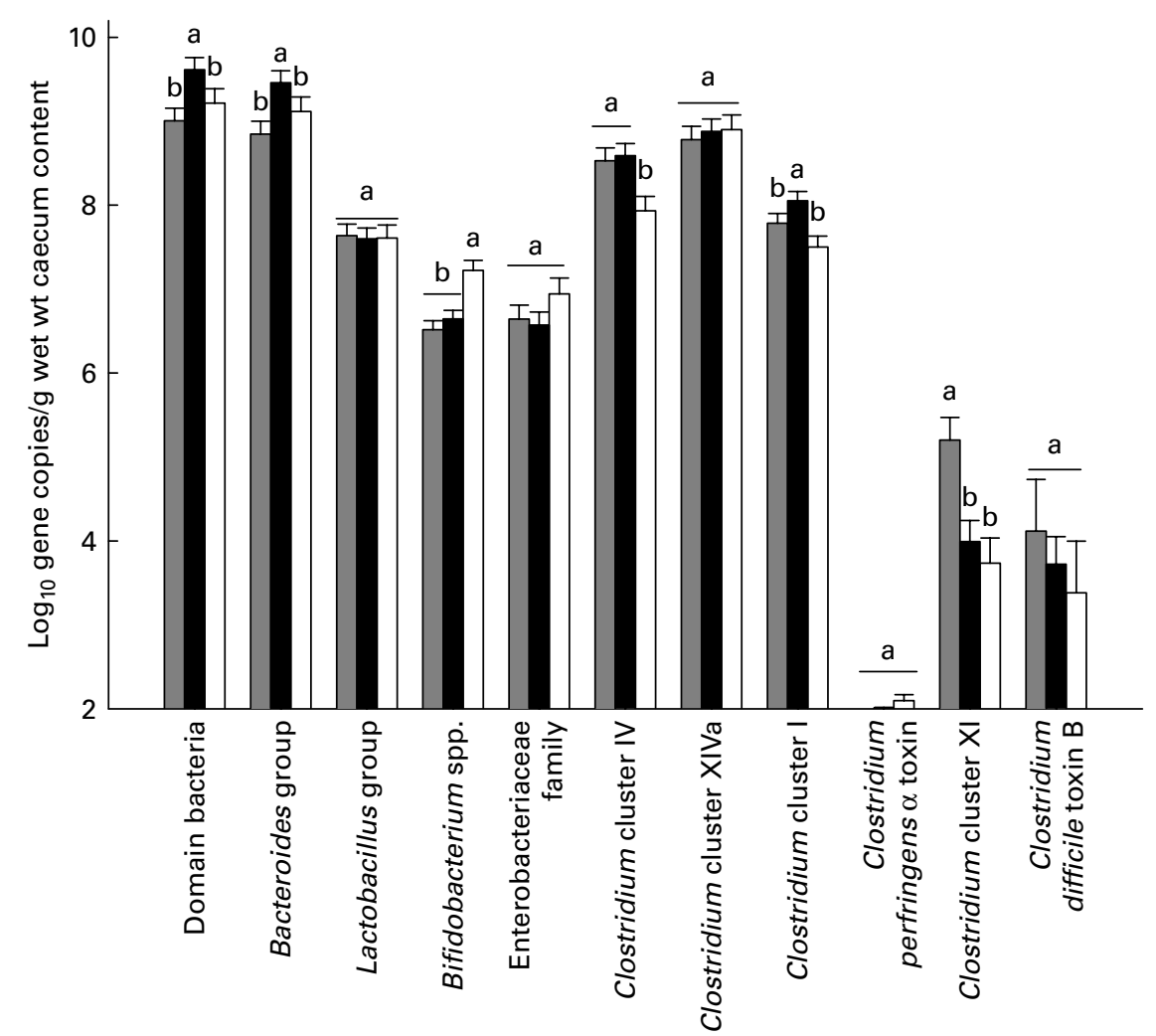

Fig. 3. Quantitative PCR analysis of bacterial populations of caecal samples ( $\square$, control; $\mathbf{\square}$, inulin; $\square$, fructo-oligosaccharides) collected from HLA-B27 transgenic rats at 16 weeks of age. Values are least-square means, with their standard errors represented by vertical bars. ${ }^{a, b, c}$ Least-square mean values (obtained with the same primer pairs) with unlike letters were significantly different $(P \leq 0.05)$. 
and the clostridial cluster IV did not segregate according to the different diets (Fig. S1, supplementary material for this article can be found at http://www.journals.cambridge.org/bjn). Amplicons obtained with primers specific for Bifidobacterium spp. migrated as a single fragment on DGGE gels, indicating the presence of one Bifidobacterium species only in each animal (data not shown). Sequence analysis of five amplicons (one FOS-fed animal, two animals each from the inulin and control groups) was performed to identify bifidobacteria on species level; four sequences matched Bifidobacterium animalis with $>99 \%$ identity; one sequence matched Bifidobacterium pseudolongum (99\% identity).

\section{Caecal and faecal SCFA}

SCFA composition in the faecal and caecal samples collected from HLA-B27 TG rats at the end point of carbohydrate treatments (16 weeks) did not differ significantly between the diets (data not shown). The total SCFA concentration of the faecal samples collected at 4 and 16 weeks of age was 54.8 (SEM 8.4) and 68.5 (sem 5.8) $\mu \mathrm{mol} / \mathrm{g}$, respectively, for the control group, 45.8 (SEM 9.4) and 78.5 (SEM 6.4) $\mu \mathrm{mol} / \mathrm{g}$, respectively, for the inulin group, and 46.7 (SEM 8.7) and $77.2($ SEM 7.4) $\mu \mathrm{mol} / \mathrm{g}$, respectively, for the FOS group. The increase in total SCFA over the treatment period was significant for inulin- and FOStreated animals $(P=0.023$ and $P=0.018$, respectively), but not for the control group. Acetate was the major product (about $70 \%$ of the total SCFA); faecal acetate significantly increased with age for all groups (data not shown).

\section{Correlations among bacterial populations, SCFA and degree of inflammation for caecum and faeces}

Correlations among the individual variables of intestinal bacterial populations, SCFA, and the degree of caecal inflammation were initially performed by PC analysis. The animals in the three treatment groups clustered separately in the linear discriminant analysis, indicating a differential effect of the two fructans on intestinal microbiota and intestinal inflammation (data not shown). Loading plots are depicted in Fig. 5(A; caecum samples) and Fig. 5(B; faecal samples) to indicate correlations between variables. In the caecal samples, a cluster located on the upper left quadrant included Clostridium cluster XI, IL-1 $\beta$ concentration, GGS, histology score, propionate and butyrate (Fig. 5(A)). Bifidobacterium spp., Enterobacteriaceae, $C$. perfringens $\alpha$ toxin, acetate and total SCFA formed clustered opposite to the first cluster, indicating a negative correlation. A third cluster containing total bacteria, Bacteroides and Lactobacillus groups and clostridial clusters I, IV, cluster XIVa was located in the upper right quadrant of the loading plot (Fig. 5(A)). In the loading plot for faecal samples (Fig. 5(B)), Bifidobacterium spp., Clostridium cluster I and $C$. perfringens $\alpha$ toxin, as well as acetate, propionate, butyrate and total SCFA formed a cluster negatively influenced by PC2. Clostridium cluster XI and $C$. difficile toxin $\mathrm{B}$ positively correlated with inflammation markers such as GGS, histology score and IL-1 $\beta$ concentration; and together formed a second cluster positively influenced by
PC2 and negatively related to the first cluster. Total bacteria, the Bacteroides and Lactobacillus groups, and the clostridial clusters IV and XIVa were separated as a third cluster, which was positively correlated mainly to PC1.

\section{Pearson correlation (0.0-100.0\%) Bacteroides group}
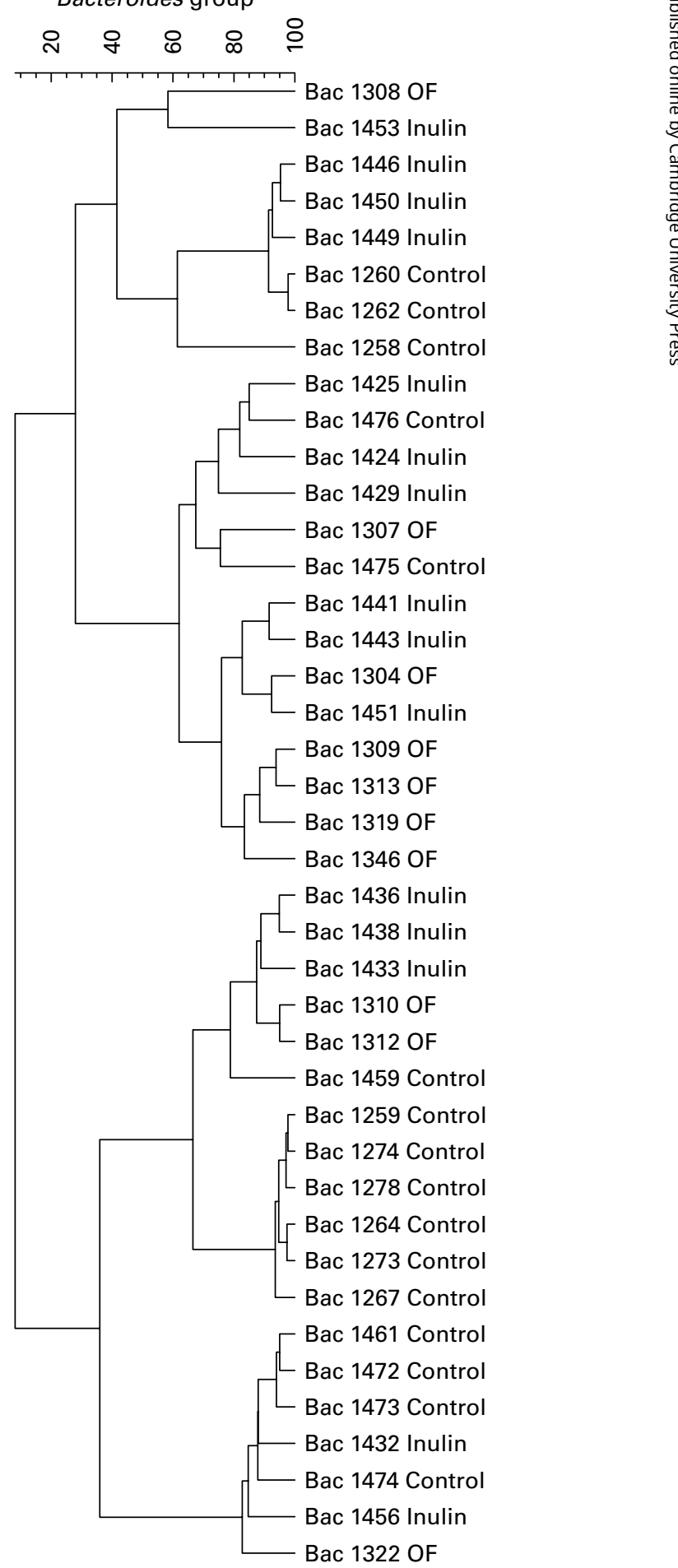

Fig. 4. Pearson correlation coefficient of denaturing gradient gel electrophoresis (DGGE) profiles of faecal communities of the Bacteroides-PrevotellaPorphyromonas group. DNA extracted from the faecal samples collected at the end point (16 weeks of age) was used as a template. 

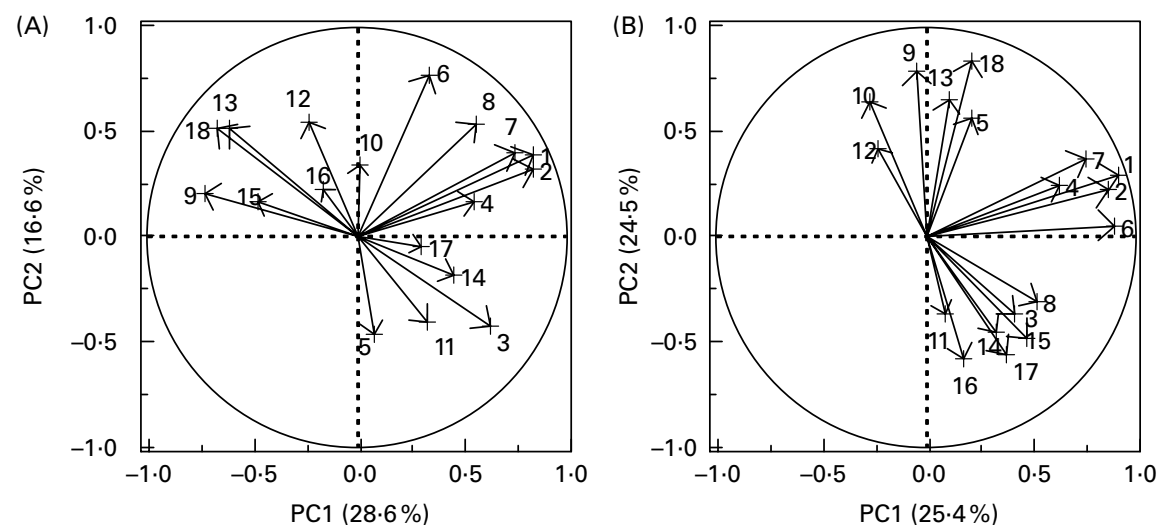

Fig. 5. Loading plots of the first two principal components (PC1 and PC2) for (A) caecum and (B) faeces show correlations among copy numbers for bacterial groups, SCFA, gross gut score (GGS) and IL-1 $\beta$ concentration. 1 - total bacteria; 2 - Bacteroides-Prevotella-Porphyromonas group; 3 - Bifidobacterium spp.; 4 - Lactobacillus-Pediococcus-Leuconostoc-Weissella group; 5 - Enterobacteriaceae family; 6 - Clostridium cluster IV; 7 - Clostridium cluster XIVa; 8 - Clostridium cluster I; 9 - Clostridium cluster XI; 10 - Clostridium difficile toxin B; 11 - Clostridium perfringens $\alpha$ toxin; 12 - IL-1 concentration; 13 - GGS; 14 - acetate; 15 - propionate; 16 - butyrate; 17 - total SCFA; 18 - histology score.

The associations between the variables were further analysed by Spearman's correlation analysis to determine which bacterial groups or metabolites were associated with chronic intestinal inflammation (Table 2). The analysis revealed significantly negative correlations of Bifidobacterium spp. with inflammation markers both in caecum and faeces. However, Clostridium cluster XI and genes coding for toxin B of C. difficile were positively correlated with inflammation (Table 2). Surprisingly, the correlations between Clostridium cluster XIVa and GGS ( $r 0.29$ and $P=0.044)$ and histology score $(r 0.39$ and $P=0.006)$ were positive.

\section{Discussion}

Dietary interventions with non-digestible fermentable carbohydrates such as inulin and FOS present a potential tool for the prevention or treatment of IBD. An understanding of their protective mechanisms of action, which probably involves an altered composition and activity of intestinal microbiota, is required in order to determine chain length, structure, and linkages type of non-digestible carbohydrates that effectively improve colitis. To our knowledge, this is the first study to determine whether dietary inulin and FOS, differing in their DP, have divergent effects on the composition of intestinal microbiota in a well-validated colitis model. Different from intervention studies using healthy animals ${ }^{(25)}$, intestinal microbiota in colitis models are also affected by intestinal inflammation ${ }^{(29)}$. In our study design, faecal microbiota were analysed over time to evaluate the effect of dietary intervention and inflammation; caecal microbiota were analysed at the time of killing of the animals to obtain insight into the composition of microbiota in the intestinal compartment exhibiting the highest degree of inflammation.

Treatment with a mixture of inulin and FOS reduced colitis in HLA-B27 TG rats ${ }^{(18)}$. Other studies in rats reported protective effects of $\beta$-fructans inulin on DSS-induced colitis ${ }^{(30)}$, and trinitrobenzene sulphonic acid-induced colitis $^{(31,32)}$. However, not all studies using fructans have reported positive outcomes ${ }^{(33)}$. A direct comparison of $\beta$-fructans differing in their molecular weight has not been reported in a colitis model. Both fructans had anti-inflammatory effects, but the effects of FOS on caecal inflammation were more pronounced compared to inulin.

Table 2. Correlations $(r)$ between abundance in bacterial groups and mucosal inflammation indicated by IL-1 $\beta$ concentration, gross gut score (GGS) and histology score in caecal or faecal samples ${ }^{*}$ (Correlation coefficients and $P$ values)

\begin{tabular}{|c|c|c|c|c|c|c|}
\hline \multirow[b]{2}{*}{ Bacterial groups } & \multicolumn{2}{|c|}{$\begin{array}{c}\mathrm{IL}-1 \beta \\
\text { concentration } \\
\text { (pg/mg protein) }\end{array}$} & \multicolumn{2}{|c|}{ GGS } & \multicolumn{2}{|c|}{ Histology score } \\
\hline & $r$ & $P$ & $r$ & $P$ & $r$ & $P$ \\
\hline \multicolumn{7}{|l|}{ Caecum } \\
\hline Bifidobacterium spp. & -0.18 & 0.278 & $-0.44 \dagger$ & 0.002 & $-0.60 \dagger$ & 0.001 \\
\hline Clostridium cluster XI & 0.22 & 0.127 & $0.53 \dagger$ & 0.001 & $0.59 \dagger$ & 0.001 \\
\hline \multicolumn{7}{|l|}{ Faeces } \\
\hline Bifidobacterium spp. & -0.15 & 0.299 & $-0.33 \dagger$ & 0.019 & -0.25 & 0.079 \\
\hline Clostridium cluster XIVa & 0.04 & 0.809 & $0.29 \dagger$ & 0.044 & $0.39 \dagger$ & 0.006 \\
\hline Clostridium cluster XI & $0.29 \dagger$ & 0.041 & $0.42 \dagger$ & 0.002 & $0.64 \dagger$ & 0.001 \\
\hline Clostridium difficile toxin B & $0.39 \dagger$ & 0.041 & $0.42 \dagger$ & 0.003 & $0.43 \dagger$ & 0.002 \\
\hline
\end{tabular}

* Correlations are assessed by Spearman's correlation test. †Coefficients with $P<0.05$ 
Prevention of chronic inflammation was previously associated with increased numbers of intestinal lactobacilli and bifidobacteria ${ }^{(18)}$. The present study confirmed that the abundance of bifidobacteria was consistently and negatively correlated with chronic intestinal inflammation. FOS but not inulin increased the numbers of caecal and faecal bifidobacteria. FOS did not alter the diversity of bifidobacteria; $B$. animalis was predominant in all animals. This divergent effect on the abundance of bifidobacteria corresponded to the differential effect of inulin and FOS on chronic intestinal inflammation and probably relates to the preferential metabolism of FOS by most Bifidobacterium spp. A majority of bifidobacteria metabolise FOS by oligosaccharide transport and hydrolysis by intracellular $\beta$-fructofuranosidases ${ }^{(15,34)}$. Only few bifidobacteria possess extracellular enzymes to degrade polymeric $\beta$-fructans such as inulin ${ }^{(34,35)}$.

Intestinal bacteria that are more abundant than bifidobacteria may also contribute to protection. FOS also increased the caecal abundance of the Bacteroides group. DGGE showed that this group, which ferments a wide range of non-digestible carbohydrates, is also qualitatively influenced by inulin and FOS. The Bacteroides group plays a controversial role in IBD. Bacteroides vulgatus induced colitis in gnotobiotic HLA-B27 TG rats ${ }^{(4,11)}$, and are found more frequently in colonic biopsies taken from UC patients compared to healthy subjects $^{(36,37)}$. However, other strains of B. vulgatus protected against $E$. coli-induced colitis in gnotobiotic IL-2-deficient mice ${ }^{(38)}$. A diet rich in resistant starch that is associated with a high faecal concentration of SCFA and a reduced risk of IBD also resulted in a high abundance of organisms in the Bacteroides group ${ }^{(39)}$.

The abundance of Enterobacteriaceae in other colitis models positively correlated with host-mediated inflammation $^{(40)}$; and pathogenic bacteria of the Enterobacteriaceae family such as pathogenic $E$. coli frequently are more abundant in IBD patients in comparison to healthy individuals $^{(8,10,29)}$. However, HLA-B27 TG rats mono-associated with E. coli did not develop colitis ${ }^{(11)}$ and E. coli Nissle 1917 even maintained remission in patients with $\mathrm{UC}^{(41)}$, indicating that some Enterobacteriaceae are protective. In this study, PC and correlation analyses of intestinal microbiota and inflammation markers did not reveal a consistent association of Enterobacteriaceae with inflammation or protection.

Chronic intestinal inflammation consistently correlated with the abundance of Clostridium cluster XI as well as the abundance of genes encoding for the $C$. difficile toxin B. Fructans substantially decreased the gene copy numbers of the Clostridium cluster $\mathrm{XI}$ in faecal microbiota. This decrease was matched by a reduction of gene copy numbers of the $C$. difficile toxin B. C. difficile is the major cause of nosocomial diarrhoea, and growth and toxin production of $C$. difficile is favoured by dysbiosis ${ }^{(42)}$. Patients with UC are prone to infections with $C$. difficile, which further increases morbidity and even mortality in UC patients who are also frequently on immunosuppressive drugs ${ }^{(43,44)}$. Independent of any colitisreducing effect of dietary $\beta$-fructans in patients, a reduction of $C$. difficile overgrowth thus justifies their therapeutic use.
SCFA - acetate, propionate and butyrate - are the major end-products of intestinal fermentation of $\beta$-fructans ${ }^{(45)}$ Lactate formation by lactobacilli and bifidobacteria increase SCFA through cross-feeding of butyrate-producing bacteria ${ }^{(46)}$. Butyrate is the main fuel for colonocytes and also promotes the down-regulation of pro-inflammatory cascades in experimental and human $\operatorname{IBD}^{(30)}$. The protective effects of bifidobacteria in an animal model for enteric infection were recently attributed to acetate formation ${ }^{(47)}$. The protective effect of a non-purified diet in DSS-induced colitis in mice when compared to a purified diet also correlated to high intestinal concentration of SCFA ${ }^{(21)}$. Stimulation of SCFA production by $\beta$-fructans may thus contribute to their antiinflammatory effect. In this study, linear discriminant indicated a negative correlation between faecal SCFA and inflammation markers. However, more than $90 \%$ of SCFA produced in the intestine are absorbed in the caecal and colonic epithelial cells ${ }^{(48,49)}$. Therefore, SCFA levels in the faeces of rats are a poor indicator of intestinal carbohydrate fermentation ${ }^{(18,25,48)}$.

In summary, despite the wide use of FOS and inulin as functional food ingredients and their well-studied prebiotic activity, very little is known about the relationship between their DP and their effect on intestinal microbiota or host health ${ }^{(25)}$. Our results demonstrate that FOS and inulin differ in colitis reduction and also vary in their effects on the intestinal microbiota in HLA-B27 TG rats. The abundance of Bifidobacterium spp. and Clostridium cluster XI correlated negatively and positively, respectively, to chronic intestinal inflammation. The protective effects of intestinal microbiota in IBD may relate to the formation of SCFA in the large intestine; however, additional studies are required to confirm a protective role of SCFA in experimental colitis and in clinical studies.

\section{Acknowledgements}

The authors wish to express their thanks to Barbara MetzlerZebeli and Prajwal Regmi for assistance in the statistical analysis. The present study was supported by Crohn's and Colitis Foundation of Canada (CCFC) and Canadian Institutes of Health Research (CIHR). P. T. K. is supported by an Interfaculty Student Scholarship of the Faculty of Medicine and the Faculty of ALES, University of Alberta. M. G. G. acknowledges the Canada Research Chair for funding. R. S. V. is a recipient of a CAG/CIHR/Abbott-Canada Fellowship Award. L. A. D. is also supported by the Alberta IBD Consortium, a grant of the Alberta Heritage Foundation for Medical Research. P. T. K., R. S. V., L. A. D. and M. G. G. designed the research; P. T. K., X. S. and R. S. V. conducted the research. P. T. K., L. A. D. and M. G. G. wrote the manuscript. All authors read and approved the final manuscript. The authors state that there are no conflicts of interest.

\section{References}

1. Hanauer SB (2006) Inflammatory bowel disease: epidemiology, pathogenesis, and therapeutic opportunities. Inflamm Bowel Dis 12, S3-S9. 
2. Farrell RJ \& La Mont JT (2002) Microbial factors in inflammatory bowel disease. Gastroenterol Clin North Am 31, 41-62.

3. Thompson-Chagoyan OC, Maldonado J \& Gil A (2005) Aetiology of inflammatory bowel disease (IBD): role of intestinal microbiota and gut-associated lymphoid tissue immune response. Clin Nutr 24, 339-352.

4. Rath HC, Herfarth HH, Ikeda JS, et al. (1996) Normal luminal bacteria, especially Bacteroides species, mediate chronic colitis, gastritis, and arthritis in HLA-B27/human beta2 microglobulin transgenic rats. J Clin Investig 98, 945-953.

5. Fichera A, McCormack R, Rubin MA, et al. (2005) Long-term outcome of surgically treated Crohn's colitis: a prospective study. Dis Col Rect 48, 963-969.

6. Frank DN, Amand AS, Feldman RA, et al. (2007) Molecularphylogenetic characterization of microbial community imbalances in human inflammatory bowel diseases. Proc Natl Acad Sci U S A 104, 13780-13785.

7. Swidsinski A, Ladhoff A, Pernthaler A, et al. (2002) Mucosal flora in inflammatory bowel disease. Gastroenterology 122, $44-54$.

8. Baumgart M, Dogan B, Rishniw M, et al. (2007) Culture independent analysis of ileal mucosa reveals a selective increase in invasive Escherichia coli of novel phylogeny relative to depletion of Clostridiales in Crohn's disease involving the ileum. ISME J 1, 403-418.

9. Sokol H, Pigneur B, Watterlot L, et al. (2008) Faecalibacterium prausnitzii is an anti-inflammatory commensal bacterium identified by gut microbiota analysis of Crohn disease patients. Proc Natl Acad Sci U S A 105, 16731-16736.

10. Thomazini CM, Samegima DA, Rodrigues MA, et al. (2011) High prevalence of aggregative adherent Escherichia coli strains in the mucosa-associated microbiota of patients with inflammatory bowel diseases. Int J Med Microbiol 301, 475-479.

11. Rath HC, Ikeda JS, Linde HJ, et al. (1999) Varying cecal bacterial loads influences colitis and gastritis in HLA-B27 transgenic rats. Gastroenterology 116, 310-319.

12. Taurog JD, Richardson JA, Croft JT, et al. (1994) The germ free state prevents development of gut and joint inflammatory disease in HLA-B27 transgenic rats. J Exp Med $\mathbf{1 8 0}$, 2359-2364.

13. Sartor RB (2004) Therapeutic manipulation of the enteric microflora in inflammatory bowel diseases: antibiotics, probiotics, and prebiotics. Gastroenterology 126, 1620-1633.

14. Roberfroid MB, Van Loo IA \& Gibson GR (1998) The bifidogenic nature of chicory inulin and its hydrolysis products. J Nutr 128, 11-19.

15. Rossi M, Corradini C, Amaretti A, et al. (2005) Fermentation of fructooligosaccharides and inulin by bifidobacteria: a comparative study of pure and fecal cultures. Appl Environ Microbiol 71, 6150-6158.

16. Campbell JM, Bauer LL, Fahey GC, et al. (1997) Selected fructooligosaccharide (1-kestose, nystose, and 1F-beta-fructofuranosylnystose) composition of foods and feeds. J Agric Food Chem 45, 3076-3082.

17. Tieking M, Kühnl W \& Gänzle MG (2005) Evidence for formation of heterooligosaccharides by Lactobacillus sanfranciscensis during growth of wheat sourdough. J Agric Food Chem 53, 2456-2461.

18. Hoentjen F, Welling G, Harmsen H, et al. (2005) Reduction of colitis in HLA-B27 transgenic rats is associated with microflora changes and immunomodulation. Inflamm Bowel Dis 11, 977-985.

19. Schultz M, Munro K, Tannock GW, et al. (2004) Effects of feeding a probiotic preparation (SIM) containing inulin on the severity of colitis and on the composition of the intestinal microflora in HLA-B27 transgenic rats. Clin Diagn Lab Immunol 11, 581-587.

20. Osman N, Adawi D, Molin G, et al. (2006) Bifidobacterium infantis strains with and without a combination of oligofructose and inulin (OFI) attenuate inflammation in DSS-induced colitis in rats. BMC Gastroenterol 6, 6-31.

21. Goto H, Takemura N, Ogasawara T, et al. (2010) Effects of fructo-oligosaccharide on DSS/induced colitis differ in mice fet nonpurified and purified diets. J Nutr 140, 2121-2127.

22. Furrie E, Macfarlane S, Kennedy A, et al. (2005) Synbiotic therapy (Bifidobacterium longum/Synergy 1) initiates resolution of inflammation in patients with active ulcerative colitis: a randomised controlled pilot trial. Gut 54, 242-249.

23. Casellas F, Borruel N, Torrejon A, et al. (2007) Oral oligofructose-enriched inulin supplementation in acute ulcerative colitis is well tolerated and associated with lowered faecal calprotectin. Aliment Pharmacol Ther 25, 1061-1067.

24. Benjamin JL, Hedin CRH, Koutsoumpas A, et al. (2011) Randomised, double-blind, placebo-controlled trial of fructo-oligosaccharides in active Crohn's disease. Gut 60, 923-929.

25. Kleessen B, Hartmann L \& Blaut M (2001) Oligofructose and long-chain inulin: influence on the gut microbial ecology of rats associated with a human faecal flora. Br J Nutr 86, 291-300.

26. Metzler-Zebeli BU, Hooda S, Pieper R, et al. (2010) Non-starch polysaccharides modulate bacterial microbiota, pathways for butyrate production, and abundance of pathogenic Escherichia coli in the gastrointestinal tract of pigs. Appl Environ Microbiol 76, 3692-3701.

27. Dmytrash A, Prajapati V, Lupicki M, et al. (2006) The prebiotic combination inulin and oligofructose reduces inflammation in the cecum and proximal colon of HLA-B27 transgenic rats. Can J Gastroenterol 20, 69A.

28. McLane BA, Uzal FA, Miyakawa MEF, et al. (2006) The enterotoxic clostridia. Prokaryotes 4, 698-752.

29. Andoh A, Sakata S, Koizumi Y, et al. (2007) Terminal restriction fragment length polymorphism analysis of the diversity of fecal microbiota in patients with ulcerative colitis. Inflamm Bowel Dis 13, 955-962.

30. Videla S, Vilaseca J, Antolin M, et al. (2001) Dietary inulin improves distal colitis induced by dextran sodium sulfate in the rat. Am J Gastroenterol 96, 1486-1493.

31. Cherbut C, Michel C \& Lecannu G (2003) The prebiotic characteristics of fructooligo-saccharides are necessary for reduction of TNBS-induced colitis in rats. J Nutr 133, 21-27.

32. Lara-Villoslada F, de Haro O, Camuesco D, et al. (2006) Short-chain fructooligosaccharides, in spite of being fermented in the upper part of the large intestine, have anti-inflammatory activity in the TNBS model of colitis. Eur J Nutr 45, 418-425.

33. Moreau NM, Martin LJ, Toquet CS, et al. (2003) Restoration of the integrity of rat caeco-colonic mucosa by resistant starch, but not by fructo-oligosaccharides, in dextran sulfate sodium-induced experimental colitis. BrJ Nutr 90, 75-85.

34. van den Broek LAM, Hinz SWA, Beidman G, et al. (2008) Bifidobacterium carbohydrases - their role in breakdown and synthesis of (potential) prebiotics. Mol Nutr Food Res 52, 146-163.

35. Korakli M, Gänzle MG \& Vogel RF (2002) Metabolism by Bifidobacteria and lactic acid bacteria of polysaccharides from wheat and rye and exopolysaccharides produced by Lactobacillus sanfranciscensis. J Appl Microbiol $\mathbf{9 2 ,}$ 958-965.

36. Matsuda H, Fujiyama Y, Andoh A, et al. (2000) Charaterization of antibody responses against rectal mucosa-associated 
bacterial flora in patients with ulcerative colitis. J Gastroenterol Hepatol 15, 61-68.

37. Lucke K, Miehlke S, Jacobs E, et al. (2006) Prevalence of Bacteroides and Prevotella spp. in ulcerative colitis. I Med Microbiol 55, 617-624.

38. Waidmann M, Bechtold O, Frick JS, et al. (2003) Bacteroides vulgatus protects against Escherichia coli-induced colitis in gnotobiotic interleukin-2-deficient mice. Gastroenterology 125, 162-177.

39. de Filippo C, Cavalieri D, di Paola M, et al. (2010) Impact of diet in shaping gut microbiota revealed by a comparative study in children from Europe and rural Africa. Proc Natl Acad Sci U S A 107, 14691-14696.

40. Lupp C, Robertson ML, Wickham ME, et al. (2007) Host-mediated inflammation disrupts the intestinal microbiota and promotes the overgrowth of Enterobacteriaceae. Cell Host Microbe 2, 119-129.

41. Kruis W, Schütz E, Fric P, et al. (1997) Double-blind comparison of an oral Escherichia coli preparation and mesalazine in maintaining remission of ulcerative colitis. Aliment Pharmacol Ther 11, 853-858.

42. Heinlen L \& Ballard JD (2010) Clostridium difficile infection. Am J Med Sci 340, 247-252.

43. Musa S, Thomson S, Cowan M, et al. (2010) Clostridium difficile infection and inflammatory bowel disease. Scand J Gastroenterol 45, 261-272.

44. DuPont AW \& DuPont HL (2011) The intestinal microbiota and chronic disorders of the gut. Nat Rev Gastroenterol Hepatol 8, 523-531.

45. Cummings JH \& Macfarlane GT (1997) Role of intestinal bacteria in nutrient metabolism. Clin Nutr 16, 3-11.

46. Louis P, Scott KP, Duncan SH, et al. (2007) Understanding the effects of diet on bacterial metabolism in the large intestine. J Appl Microbiol 102, 1197-1208.

47. Fukuda S, Toh H, Hase K, et al. (2011) Bifidobacteria can protect from enteropathogenic infection through production of acetate. Nature $\mathbf{4 6 9}, 543-547$.

48. Regmi PR, Metzler-Zebeli BU, Gänzle MG, et al. (2011) Starch with high amylose and low in vitro digestibility increases intestinal nutrient flow and microbial fermentation and selectively promotes bifidobacteria in pigs. J Nutr 141, $1273-1280$.

49. Topping DL \& Clifton PM (2001) Short-chain fatty acids and human colonic function: roles of resistant starch and non-starch polysaccharides. Physiol Rev 81, 1031-1064.
50. Lee DH, Zo YG \& Kim SJ (1996) Nonradioactive method to study genetic profiles of natural bacterial communities by PCR-single-strand-conformation polymorphism. Appl Environ Microbiol 62, 3112-3120.

51. Walter J, Hertel C, Tannock GW, et al. (2001) Detection of Lactobacillus, Pediococcus, Leuconostoc, and Weissella species in human feces by using group-specific PCR primers and denaturing gradient gel electrophoresis. Appl Environ Microbiol 67, 2578-2585.

52. Heilig HG, Zoetendal EG, Vaughan EE, et al. (2002) Molecular diversity of Lactobacillus spp. and other lactic acid bacteria in the human intestine as determined by specific amplification of $16 \mathrm{~S}$ ribosomal DNA. Appl Environ Microbiol 68, 114-123.

53. Rinttila T, Kassinen A, Malinen E, et al. (2004) Development of an extensive set of $16 \mathrm{~S}$ rDNA targeted primers for quantification of pathogenic and indigenous bacteria in faecal samples by real-time PCR. J Appl Microbiol 97, 1166-1177.

54. Bartosch S, Fite A, Macfarlane GT, et al. (2004) Characterization of bacterial communities in feces from healthy elderly volunteers and hospitalized elderly patients by using real-time PCR and effects of antibiotic treatment on the fecal microbiota. Appl Environ Microbiol 70, 3575-3581.

55. Matsuki T, Watanabe K, Fujimoto J, et al. (2004) Use of $16 \mathrm{~S}$ rRNA gene-targeted group-specific primers for real-time PCR analysis of predominant bacteria in human feces. Appl Environ Microbiol 70, 7220-7228.

56. Matsuki T, Watanabe K, Fujimoto J, et al. (2002) Development of 16S rRNA-gene-targeted group-specific primers for the detection and identification of predominant bacteria in human feces. Appl Environ Microbiol 68, 5445-5451.

57. Song Y, Liu C \& Finegold SM (2004) Real-time PCR quantitation of clostridia in feces of autistic children. Appl Environ Microbiol 70, 6459-6465.

58. Messelhäusser U, Zucker R, Elmer-Englhard D, et al. (2007) Nachweis und Charakterisierung von Clostridium perfringens mittels real-time PCR (Detection and characterization of Clostridium perfringens by real-time PCR). J Verbr Lebensm 2, 194-197.

59. van den Berg RJ, Kuijper EJ, van Coppenraet LE, et al. (2006) Rapid diagnosis of toxinogenic Clostridium difficile in faecal samples with internally controlled real-time PCR. Clin Microbiol Infect 12, 184-186. 